Objective to investigate the association between body mass index (BMI) and fruit juice intake in Brazilian adults.

Method The Telephone Survey System for the Surveillance of Risk and Protective Factors for Chronic Non-Communicable Diseases (VIGITEL) is conducted every year since 2006. In 2008 was added a question regarding fruit juice intake. We analysed 2008 and 2009 data including 89.841 adults (20-65 years) from 27 Brazilian cities investigated. Weighted regression analyses included sex, age (years), schooling (years), level of physical activity (active/inactive) as independent variables, and BMI $\left(\mathrm{kg} / \mathrm{m}^{2}\right)$ as the dependent variable. Eating habits were evaluated based on the daily frequency of intake of fruit juices, fruits, vegetables and sugar-sweetened soft drinks.

Results Fifty-three percent of the participants were female, mean age was 37.2 years, mean BMI was $25 \mathrm{~kg} / \mathrm{m}^{2}$, and $19 \%$ reported a daily intake of fruit juice. BMI was negatively and significantly associated with fruit juice intake $(B=-0.29$; $p$-value $<0.0001$ for women; $B=-0.23$; $p$-value $=0.0004$ for men). There is a small negative association of fruit juice intake with soft drinks consumption and inactivity. After adjustment for sex, age, schooling, level of physical activity, intake of fruits, vegetables and soft drinks this association remained statistically significant $(~ B=-0.10$; $p$-value $=0.03$ ).

Conclusion Among Brazilian adults fruit juice intake may be a marker of healthier eating habits.

\section{P2-60 FREQUENCY OF OUT-OF-HOME EATING AND DIETARY HABITS IN THE BRAZILIAN TELEPHONE-BASED SURVEILLANCE SYSTEM}

doi:10.1136/jech.2011.142976h.95

${ }^{1}$ I N Bezerra, ${ }^{2} \mathrm{~A}$ de Moura Souza, ${ }^{*} \mathrm{C}$ A Monteiro, ${ }^{1,2} \mathrm{R}$ Sichieri. ${ }^{1}$ Department of Clinical and Experimental Medicine, University of the State of Rio de Janeiro, Rio de Janeiro, Rio de Janeiro, Brazil; ' $D$ Department of Epidemiology, Institute of Social Medicine, University of the State of Rio de Janeiro, Rio de Janeiro, Rio de Janeiro, Brazil; ' ${ }^{3}$ Department of Nutrition, School of Public Health, University of Sao Paulo, São Paulo, São Paulo, Brazil

Introduction The proportion of food purchased for out-of-home eating in Brazil has been increasing.

Objective To evaluate dietary habits associated with out-of-home eating among Brazilian adults.

Methods We analysed data of 135249 adults (20-65 y), living in 27 Brazilian cities, from the 2007, 2008 and 2009 Telephone Survey System for the Surveillance of Risk and Protective Factors for Chronic Non-Communicable Diseases (VIGITEL). Frequency of outof-home eating was based on the reported frequency of consuming lunch and dinner away from home per week. Dietary habits were based on the reported consumption of visible fat in meat and chicken and the daily consumption of soft drink, fruit and milk. Frequencies were weighted and analysis incorporated the complex design of the survey.

Results Men reported eating out more frequently than women (52.2\% vs $39.4 \%$ for at least once per week). Consumption of visible fat and soft drink increased with the frequency of out-of-home eating $(p<0.008)$, whereas daily fruit and milk consumption decreased $(p<0.01)$. After controlling for age and years of schooling, the OR of consuming fruit and milk decreased with the frequency of out-of-home eating ( $\mathrm{OR}=0.76$ (95\% CI 0.68 to 0.86$), \mathrm{OR}=0.79(95 \%$ CI 0.70 to 0.90 ) among men and $\mathrm{OR}=0.69$ (95\% CI 0.62 to 0.76 ), $\mathrm{OR}=0.76(95 \%$ CI 0.69 to 0.84$)$ among women, respectively, comparing 5 or more times/week with no out-of-home eating).

Conclusion Frequency of out-of-home eating was positively associated to negative markers of dietary habits and negatively associated to healthy markers of dietary habits among Brazilian adults. Due to the increasing out-of-home eating, public health strategies should be developed.

\section{P2-61 CHILDHO0D OBESITY AND PARENTAL SMOKING AS RISK FACTORS FOR CHILDHOOD ADHD IN LIVERPOOL CHILDREN}

doi:10.1136/jech.2011.142976h.96

${ }^{1} \mathrm{G}$ Koshy, ${ }^{2} \mathrm{~A}$ Delpisheh, ${ }^{* 1} \mathrm{~B}$ Brabin. ${ }^{1}$ Child and Reproductive Health Group, Liverpool School of Tropical Medicine, Liverpool, UK; ${ }^{2}$ Epidemiology Department, Ilam University of Medical Sciences, Ilam, Iran

Introduction $\mathrm{ADHD}$ prevalence has risen in parallel with rising prevalence of pregnancy smoking and childhood obesity. The objective was to determine the epidemiological association of pregnancy smoking and childhood obesity with ADHD.

Methods A cross-sectional community study was conducted in 2006 using a parental questionnaire. A total of 1074 schoolchildren aged 5-11 years were enrolled from 15 primary schools in a lower socioeconomic area of Merseyside. ADHD was defined by the question "does your child have Attention Deficit Hyperactivity Disorder, (ADHD), which has been diagnosed by a doctor?"

Results The prevalence estimates for childhood obesity, maternal smoking during pregnancy and childhood ADHD were 14.9\% (116/ 777), 28.0\% (269/955), and 3.4\% (32/945), respectively. ADHD prevalence was increased in children with obesity (RR $4.80,95 \% \mathrm{Cl}$ 2.2 to $10.4, \mathrm{p}<0.001$ ) and in children of mothers who smoked during pregnancy (RR $2.44,95 \%$ CI 1.2 to $4.9, \mathrm{p}=0.02$ ). Regression analysis adjusting for obesity, overweight, maternal smoking during pregnancy, heavy maternal smoking, household member smoking during pregnancy, doctor-diagnosed asthma, preterm birth, and low birthweight showed significant independent associations of ADHD prevalence with obesity (AOR 4.66, 95\% CI 1.57 to $13.89, p=0.006$ ) and pregnancy smoking (AOR 3.19, 95\% CI 1.08 to 9.49, $\mathrm{p}=0.04$ ). There was a positive dose-response association of $\mathrm{ADHD}$ with the number of maternal cigarettes smoked during pregnancy.

Conclusion Measures to reduce both smoking among pregnant women and childhood obesity might reduce prevalence of childhood $\mathrm{ADHD}$.

\section{P2-62 EARLY DETECTION OF COLORECTAL CANCER IN THE US IN APPALACHIA}

doi:10.1136/jech.2011.142976h.97

M Dignan, ${ }^{*}$ B Shelton, K Pearce, N Schoenberg, C Tolle, S Slone. University of Kentucky, Lexington, Kentucky, USA

Colorectal cancer is currently the $3^{\text {rd }}$ leading cause of cancer death worldwide. For rural, medically underserved populations in the US, and particularly in Appalachia, the burden of this disease is even greater. Elevated colorectal cancer rates in rural populations represents a missed opportunity for early detection through screening. Low screening rates are particularly concerning for Appalachia because of poverty and limited access to healthcare. To address this problem, a 5 -year study to increase screening through outreach to rural primary care providers was conducted from 2005 to 2010. Practices were randomised to early or delayed intervention groups and an intervention was provided in personal visits at the practices. The intervention was designed to assist practices in identifying opportunities to increase screening by providing education regarding screening efficacy, clinical performance measures, patient counselling, and creating a screening-friendly practice environment. Evaluation data were collected through medical record reviews before and after intervention delivery. Baseline data for both study groups indicated that healthcare providers had completed screening by any method in $36.7 \%$ of the 3906 patient records patient records reviewed. There was no significant difference in screening rates between the early or delayed group practices at baseline. At followup, screening rates remained low overall, $37.9 \%$. However, for practices that received the intervention, analyses of follow-up data 